\title{
Margin Uninvolved by Sarcoma
}

National Cancer Institute

\section{Source}

National Cancer Institute. Margin Uninvolved by Sarcoma. NCI Thesaurus. Code C159235.

The marg in of the sample has no sarcoma. 